\title{
New atmospheric profiles for H.E.S.S. data analysis
}

\author{
Mónica Seglar-Arroyo ${ }^{1, \star}$ and Fabian Schüssler ${ }^{1, \star \star}$ on behalf of the H.E.S.S. Collaboration \\ ${ }^{1}$ IRFU, CEA Saclay
}

\begin{abstract}
.
Particles arriving to Earth allow us to scan and improve our understanding of the Universe, both in its composition and its dynamics. For ground-based experiments in astroparticle physics, the atmosphere plays a key role and its understanding, monitoring and modelling is essential for a realistic description in data analysis and simulations. In this work we present the development of a novel experimental atmospheric model, the GNNA120 model, describing the atmosphere above the H.E.S.S. gamma-ray observatory in Namibia. This new description is the most realistic to date and introduces the possibility to study variations at different time scales. This enables us to provide the first ever study of seasonal effects based on actual measured atmospheric profiles for the H.E.S.S. observatory and opens up the window to further improvements of the H.E.S.S. Monte Carlo simulations.
\end{abstract}

\section{Introduction}

Much of the radiation propagating in the Cosmos and incident on the Earth is thermal radiation generated in hot objects such as stars. However, it is well-known that certain particle populations in the Universe cannot result from thermal processes, but they must be produced by collective mechanisms. The best-known of this type of population are cosmic rays.

Even though the existance of cosmic rays has been known since the first decades of the $20^{\text {th }}$ century, their acceleration locations have not been found yet. In fact, deflections of charged particles in galactic and/or extragalactic magnetic field are so large that over most of the energy regime, the pointing information is completely lost; only at the very highest energies, where the energy lowers the deflection effect, in the domain of the AUGER experiment, the directional information can possibly be exploited. In this context, non charged particles neutrinos and photons play a key role to study high-energy cosmic rays and their sources.

The detection of gamma-rays can be done on board of a satellite, e.g. Fermi-LAT or AMS, or at ground level, e.g. Veritas, MAGIC and H.E.S.S., depending on the energy range. The atmosphere acts as an active target in groundbased experiments, since it is the target medium for the cosmic particle, but also the emitter of Cherenkov photons and the transport medium for those photons. Being a continuously changing system, constantly monitoring and correct parametrisation of the atmosphere plays a crucial role. Varying atmospheric conditions in terms of state variables may alter the development and detection of extensive air

\footnotetext{
^e-mail: monica.seglar-arroyo@cea.fr
}

$\star \star$ e-mail: fabian.schussler@cea.fr showers. The main goal of the study presented here is to revise the H.E.S.S. atmospheric parametrisation and quantify the systematic errors from an incorrect description.

The paper is structured as follows: In $\S 2$, the characterisation of the atmosphere above the H.E.S.S. site is presented. Following this, the new experimental GNNA120 atmospheric model is introduced and explained. In $\S 3$, we present and compare the former and new atmospheric profile and other atmospheric features. The results are discussed and summarized in $\S 4$.

\section{Characterising the atmosphere above H.E.S.S.}

\subsection{Previous studies of the atmosphere above H.E.S.S.}

H.E.S.S. is a stereoscopic system consisting of five imaging atmospheric Cherenkov telescopes located at 1800 meters above sea level in the Khomas highlands of Namibia (2316' 18" South, $16^{\circ} 30^{\prime}$ 00" East). The four H.E.S.S. phase I telescopes are arranged in a square of 120 side length, with the large H.E.S.S. II telescope at its centre.

In the H.E.S.S. experiment, the gamma-rays are detected indirectly via the Cherenkov light emitted by the charged particles of the induced air-shower. Thus, a good understanding of the atmosphere that acts as the calorimeter of the system is crucial for the Imaging Atmospheric Cherenkov Technique (IACT) method.

In 1999 , motivated by the advent of a new generation of gamma-ray observatories, atmospheric density profiles, as well as several light absorption and scattering processes dependency on geographic position and time variability were investigated by Konrad Bernlöhr [1]. The most important aspects treated in the mentioned work are: 
- Vertical structure of the atmosphere. Being the most influential parameters, it was seen that different density profiles lead to differences in Cherenkov light density up to $60 \%$. Concretely, seasonal variation at mid-latitude sites were found to be of the order of $15-20 \%$ as can be seen in Figure 1 where several average lateral distributions of Cherenkov light photons have been simulated with CORSIKA 5.71 [2] taking into account absorption of Cherenkov light.

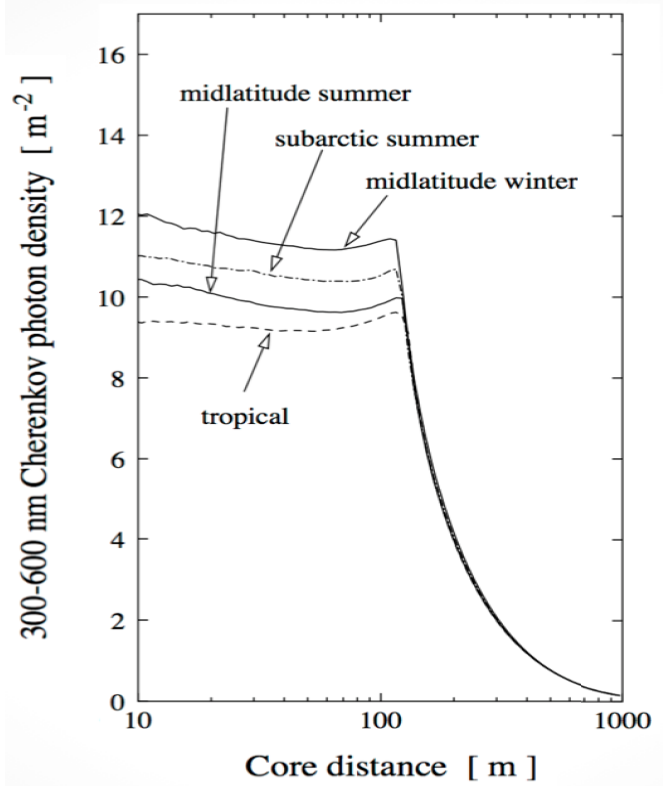

Figure 1: Average lateral distribution of Cherenkov light photons in the wavelenght range $300-600 \mathrm{~nm}$ for vertical $100 \mathrm{GeV}$ gamma-ray showers in CORSIKA $5.71 \mathrm{sim}$ ulations with different atmospheric profiles (200 showers) from [1]

- Atmospheric extinction of Cherenkov light. It includes absorption and scattering of the electromagnetic radiation by dust and gas between the emitter and the observer. For those phenomena, the main concern is that extrapolation for high-altitudes must be avoided, and monitoring procedures must be applied even though a measurement of the aerosol vertical structure is rather difficult. However, Lidar-based methods are available to measure the extintion profile with a $10 \%$ accuracy.

Note that within the H.E.S.S. data analysis and simulation tools, the Cherenkov transparency coefficient [3] takes into account the presence of elevated aerosols concentrations and of large-scale clouds. The parameters that are used as an input for its calculation are the energy threshold of the telescope, which shows a direct proportion to the transparency of the atmosphere, and other inversely proportional quantities related to the telescope data taking efficiency like the telescopes-wise muon efficiency, average pixel gains and number of active telescopes. This approach has been proven to be an effective and a pratical method of distinguinshing good quality datasets without the need to measure the aerosols distributions [3] , that is a rather difficult task.
- Refraction.The wavelenght independency of the refractive index was assumed since $\mathrm{n}(\lambda)-1$ changes only $5 \%$ over the wavelenght range $300-600 \mathrm{~nm}$, which is the range typically covered by photomultipliers. The influence on gamma-ray observations can therefore be considered as negligible [1].

The vertical atmospheric profiles used within KASKADE [4], one of the two main simulation frameworks used within the H.E.S.S. collaboration, was derived from balloon soundings at Windhoek airport in the 1990s, averaged over a whole year. A verification of this parametrisation is the main aim of the work presented here.

\subsection{Experimental GNNA120 atmospheric model}

Worldwide, the atmosphere is being continuously monitored by a fleet of satellites, networks of weather stations, ballon soundings, etc. Here we exploited different databases belonging to NOAA and NASA's monitoring and modeling centers, respectively. A full atmospheric profile model, named experimental GDAS - NRLMSISE for Namibian Atmosphere up to $120 \mathrm{~km}$ (GNNA120) model is introduced. For this new description of the atmosphere above H.E.S.S. two different sources of data have been chosen.

- Low altitudes. The Global Data Assimilation System [5] (GDAS) is a system used by the National Center for Environmental Prediction (NCEP) Global Forecast System (GFS) to place observations into a gridded model space for the purpose of starting weather forecasts with observed data. The full GDAS data are publically available via the NOAA National Operational Model Archive and Distribution System (NOMADS) [6] which is a Web-services based project providing both real-time and retrospective access to climate and weather model data.

GDAS adds various types of observations to a gridded, 3-D, global model: surface observations, ballon data, wind profiler data, aircraft reports, buoy observations, radar observations, and satellite observations. Based on simulations and weather predictions tied to measurements model data is available with a time resolution of 3 hours. The provided data contain information of 23 different levels, up to an altitude of about 25 kilometers above the surface. The levels are related to the pressure at the layer and span a range from $1000 \mathrm{hPa}$ to $20 \mathrm{hPa}$. The GDAS grid spans the whole Earth with a grid-size is of $1^{\circ} \times 1^{\circ}$ in longitude and latitude. The data available in every dataset are temperature, pressure, geopotential height, u-wind, v-wind, vertical pressure velocity and relative humidity.

The coordinates for the HESS II experiment $\left(23.27^{\circ} \mathrm{S}\right.$, $16.5^{\circ} \mathrm{E}$, and $1800 \mathrm{~m}$ ASL, Namibia) while the closest station that launches weather ballons is located at the Windhoek airport $\left(22.57^{\circ} \mathrm{S}, 17.10^{\circ} \mathrm{E}\right.$, and $1700 \mathrm{~m}$ ASL, Namibia).

- High altitudes. The Community Coordinated Modeling Center (CCMC) provides several empirical models. 
The NRLMSISE-00 Model 2001 [7] describes the neutral temperature and densities for the Earth's atmosphere up to thermospheric heights. It includes temporal and spatial variatons at various scales based on an analytical model.

The profiles that could be extracted from GDAS only present data up to around $25 \mathrm{~km}$. Thus, once one runs out of data from the GDAS database, the data is taken from the NRLMSISE-00 model up to 120 kilometers, in layers of 5 kilometers. It has been verified that both models agree very well in the overlapping region, thus no artefacts are induced by this combination.

\section{Results}

\subsection{GNNA120 atmospheric profiles}

Temperature and density profiles for the combined data set extracted from GDAS and NRL-MSISE are shown in Figure 2. One can appreciate the expected changes of the profiles while increasing the altitude, from troposphere up to thermosphere, going through stratosphere and mesosphere. The steep changes in temperature correspond to the pauses between these layers.
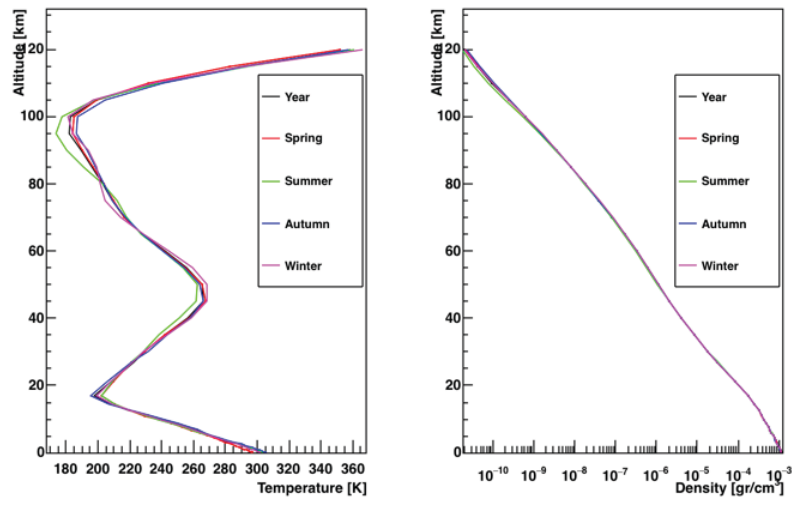

Figure 2: (a) GNNA120 yearly and seasonal averaged temperature profiles up to $120 \mathrm{~km}$ (b) Yearly and seasonal averaged density profiles up to $120 \mathrm{~km}$.

In order to check the impact of the humidity on the density profiles, the comparison for the averaged density profiles while taking into account humidity effects is plotted in Figure 3 for the different season and the whole year. For computing the humidity, a very accurate formula for determining the saturation vapor pressure by Herman Wobus in [8] has been used. The relative changes have a maximum value of $0.7 \%$, what lead us to the conclusion that this effect is rather small compared to other systematics.

Note that the humidity is an important parameter for fluorescense techniques and that in the case of condensed water, it is currently taken into account into the transparency coefficient. It can also be noted that in future experiences as CTA, it would be measurable by Raman LIDARs, and included in aerosols simulations.

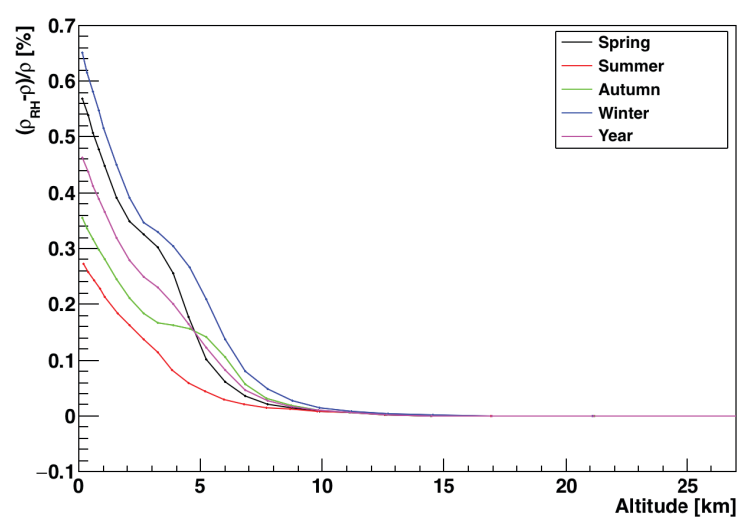

Figure 3: Difference in percentage of the density averaged profile when taking into account humidity effects

\subsection{Comparison KASKADE-to-GNNA120 atmospheric profiles}

In order to compare the former and the new GNNA120 atmospheric profiles, relative residuals have been computed and shown in Figure 4.
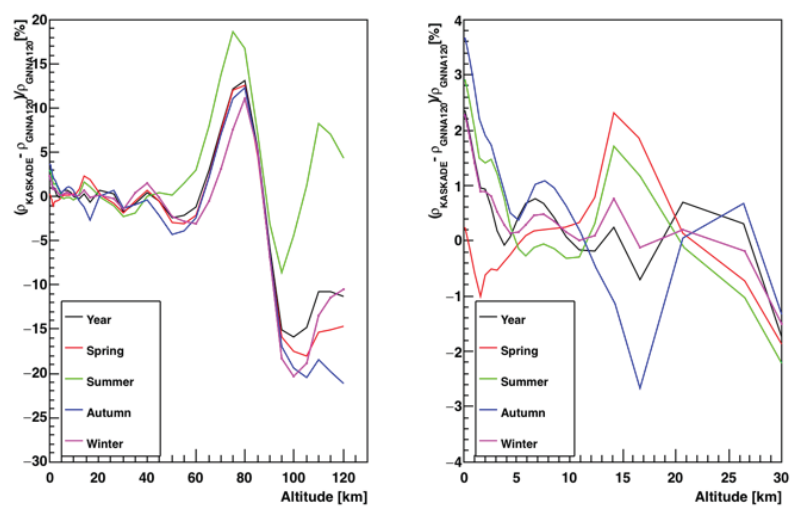

Figure 4: (a) Residuals of the computed GNNA120 model and the H.E.S.S. profiles used for simulations (b) Residuals zoomed at the ROI, important for the gamma-ray induced air shower developement.

The biggest changes (up to 20\%) are found in the upper regions of the atmosphere. However, the region of interest (ROI) important for the development of $\mathrm{TeV}$ gamma-ray induced air showers ranges from an altitude of $2 \mathrm{~km}$ to 26 $\mathrm{km}$. There, the largest differences, up to $4 \%$ from the old KASKADE profile, are given for the seasonal GNNA120 profiles, namely for spring and autumn, around altitudes of 15-20 kilometers. Comparing KASKADE to the yearly GNNA120 profile, the changes are less significant and do not exceed $1 \%$.

\subsection{Slant depth}

In principle, one can assume that the big changes up to $20 \%$ seen in Figure 4 for the density in the upper layers of the atmosphere will not change the development of the shower in simulations, since the density goes rapidly to 
zero as one increases to these altitudes. Seeking to test that assertion, we calculate the cumulative slant depth since it gives an estimation of the changes of the place in the atmosphere where the first interaction takes place and the following air shower evolution. The yearly-seasonal comparison is shown in Figure 5 where the biggest changes up to $3 \mathrm{~g} / \mathrm{cm}^{2}$ are visible for autumn and spring.

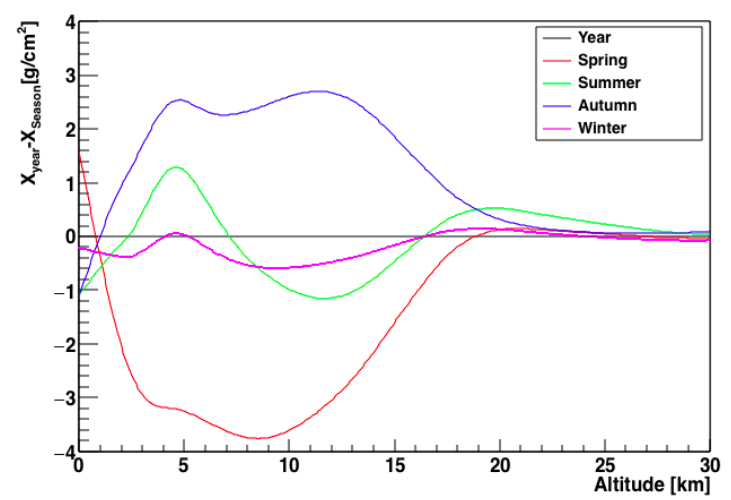

Figure 5: Differences between seasonal averaged and yearly averaged slant depth as a function of the altitude in the ROI. The altitude range was set to the ROI since for high altitudes, it goes rapidly to zero.

We note that the size of the effect is small compared to the intrinsic shower-to-shower fluctuations.

\section{Summary}

The impact of the parametrisation of different atmospheric variables relevant for the atmospheric Cherenkov technique in the atmosphere above the H.E.S.S. site has been presented by means of data coming from GDAS and NRLMSISE, the new GNNA120 model has been built.

The atmospheric profile used in the H.E.S.S. analysis, namely a yearly average at four different altitudes with interpolated values for the density up to $120 \mathrm{~km}$, has been compared to the new GNNA120 model. In the ROI, the yearly comparison to the KASCADE profile shows differences of about $1 \%$, meaning that the implementation of the yearly averaged atmosphere in the H.E.S.S. analysis is fully acceptable. This implies that, overall, we found surprisingly small differences with the 20 year old implementaton in KASKADE and, thus, our study can therefore be considered as a validation of the profiles used in the H.E.S.S. analyses so far.

Note that the GNNA120 model including seasons, developed during this work, has been included in the HESS analysis software. Results show up to a $20 \%$ of difference and the following air shower evolution at high altitudes and up to $3 \%$ for the seasonal profiles in the important range for the gamma-ray induced air shower development. This emphasizes the importance of including seasonal changes and encourages us to further study effects at even shorter timescales.

The possibility of including humidity effects into the density profiles has been studied. The effect never exceeds $1 \%$ in any of the studied cases. Despite this fact, it is a parameter that presents huge spatial and temporal fluctuations. Thus, we decided to not take it into account in the average profiles.

Some changes with respect to the yearly slant depth profile have been found for autumn and spring. The scale of these $(<4 \mathrm{~g} / \mathrm{cm} 2)$ is modest and should not significantly influence the air shower simulations.

After checking the impact of the seasonal changes, one wants to go one step further by taking into account smallscale effects. The presented study opens up the window to further implementations of the parameters that describe the chaotic nature of the calorimeter of our experiment, the atmosphere. The next step of the GNNA120 model and, indeed, the ultimate goal would be to implement atmospheric profiles every 3 hours a new method for Monte Carlo simulations: : simulations reproducing the actual data taking conditions in a time dependent fashion, so called Run-Wise Simulations currently under development within the H.E.S.S. Collaboration.

\section{References}

[1] Bernlohr, Astroparticle Physics 12 (4), 255-268 (2000)

[2] D. Heck, J. Knapp, J. Capdevielle, G. Schatz, T. Thouw (https://www.ikp.kit.edu/corsika/) Wissenschaftliche Berichte, Forschungszentrum Karlsruhe FZKA 6019 (1998)

[3] Hahn, Joachim and de los Reyes, R, EPJ Web of Conferences 89, 02002 (2015)

[4] M.P. Kertzman and G.H. Sembroski, NIM A 343, 629 (1994)

[5] Global Data Assimilation System, https://www.ncdc. noaa.gov

[6] NOAA National Operational Model Archive and Distribution System, http://nomads.ncdc.noaa.gov/

[7] Community Coordinated Modeling Center, http:// ccmc.gsfc.nasa.gov/modelweb/atmos/nrlmsise00.html

[8] Wahiduddin, https://wahiduddin.net/calc/density_ altitude.htm 